\title{
Research of Multi-level Context-aware Control Model Based on Event-driven method
}

\author{
Dongjuan Xue \\ School of Mechanical and Power Engineering, Dalian Ocean University, Liaoning, China \\ xuedongjuan@dlou.com
}

Keywords: Dynamic data model, Event-driven, Multi-level context-aware

Abstract. Context-aware applications can sense and explore the context of production processes to provide proper and useful services to managers in workshop. Firstly, a multi-level ontology model of materials is found which is expressed in OWL as an ontology collection of describing places ages and events and associated properties among them. Secondly, a multi-level context-aware control model and its mechanism are produced. In this model, data integration is controlled by the demand state transitions at different levels including workflow levels, control levels and data levels, which incorporates dynamic context information. And context is classified based on the analysis of demand into five main categories, which is further categorized as simple condition and composite condition to form a multi-level context. And the data integration model is implemented dynamically by checking the context constraint associated with each application task at the time of the specific request submission. Finally, a case study is developed within a manufacturing enterprise to show the benefits of the model.

\section{Introduction}

Because of complicated manufacturing, different production cycles of its components and parts and uncertain handicraft routes of complex equipment products, the study on materials data model and data control has strong actual significance during the production process of complex equipments. Workshop is the crucial location among the whole enterprise production activities. And at the same time material supplies are the basis and prerequisite guarantee for workshops carry out their productions, so the monitored key activities should be carried out emphasizely among workshops.

An important step to achieve this object is the integration of intelligent agents that reasoning to understand the local context and share this information in the workshop to support the intelligent applications within decision system. We are developing a new architecture supporting context-aware systems in smart environment which likes assemble lines. By context, we mean information about a location, its environmental attributes (e.g. Equipment, Operator, temperature and time) and devices, objects and software agents. Context may also include system capability, services offered and sought, the activities and tasks in which people and computing entities are engaged, and their situational roles, beliefs, and intentions.

\section{Definition and partition of multi-level ontology model}

According to the difference of materials production and their controlling requirements, this article has defined 4-level monitoring model. Those are product monitoring, inventory monitoring, component monitoring and procedure monitoring. And at based on the importance of components and parts to the end product, if this part is a key component to the end product, the monitoring and tracking degree is divided into two kinds of types: emphasizing monitoring and normal monitoring.

The capability of normal monitoring is to supervise the general production process of a component or part. While emphasizing monitoring must supervise working procedure of each activity, including monitor production route of normal component and each procedure of key component and part. Multi-level materials monitoring model is shown as Fig.1. 


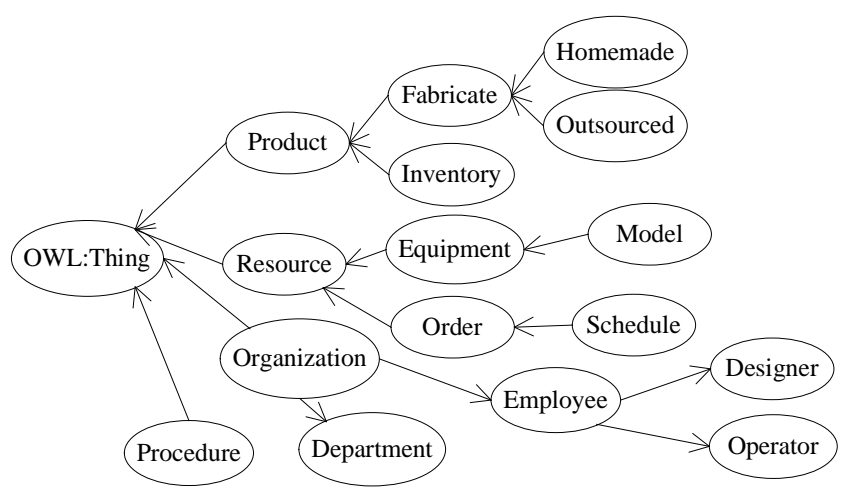

Fig.1. Multi-layer network of production monitoring

Product Monitoring. Its duty is to supervise the status which has been completed of the end product and it is carried out by coordination with plans and the information retailing into the storage of each finished product in visual inventor. It also applies to the supervision to all components and parts, including emphasizing monitoring for those key components and parts and normal monitoring for general ones.

Inventory Monitoring. Its duty is to supervise the concrete information that is retailed into and purchased out of the storage during the production process within the workshop or branch factory.

This is to come true through picking up the information such as workpiece retailed into and purchased out of the storage, semi-finished product retailed into and purchased out of the storage, finished product retailed into the storage, and so on. It applies emphasizing monitoring mode on key components and parts mainly.

Procedure Monitoring. The duty is to supervise each working procedure of key components and parts that are been manufactured exactly. This monitoring mode is suitable to emphasizing monitoring on key parts and cannot gain finished information of each procedure from visual inventory while to build up procedure account of parts according to every promised procedure in each workshop or branch factory, then track back corresponding work-ticket information to working procedure account within workshop or branch factory.

Component Monitoring. Here the components are those that produced by some workshop or branch factory bearing its contract. That is, both of the sub-component or sub-part of this component are produced and supervised only by whose corresponding workshop or branch factory.

\section{Multi-level Context-aware Control Mechanism}

Material data integration process needs dynamic and real-time decisions based on application context, it is sufficiently powerful to enforce rules and constrains that are dependent on run-time parameters, in order to take into account the contextual information and thus being able to adapt itself based on the current conditions. Thus, context has to be incorporated in the data integration mechanism, in order to extend the traditional material data integration model to gain many advantages from its context-aware capability.

Simple context condition is a predicate that checks the validity of a condition and consists of an operator and two operands. The first operand is context data, while the second one may be either context data or a predefined value. The simple context condition returns a Boolean value (true or false). Actually, this is the mechanism for obtaining first-order context information from raw material data.

Composite context condition is defined as a regular expression, consisting of Boolean operators and two or more operands. These operands may be simple context conditions and context data of Boolean type. Note that, by its definition, a composite context condition may contain other composite context conditions, as well.

Fig.2 illustrates a material data integrating control with context-aware, which is divided into three levels including workflow level, control level and data level concerning to multi-level context. 


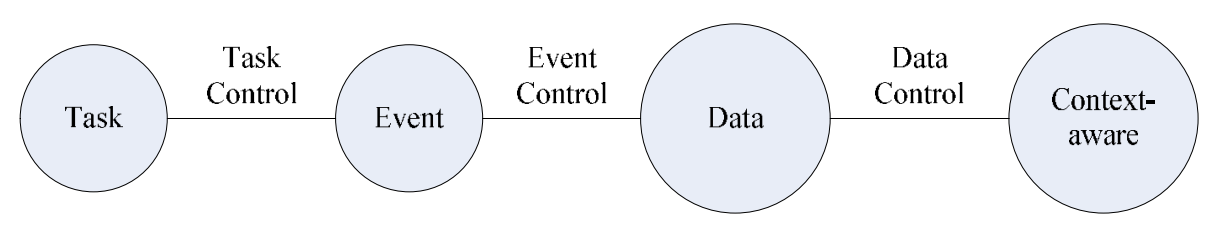

Fig.2. The Multi-levels of data integration

These categories are analyzed as follows: with respect to the workflow level, the level consists of related authorization processes of task that correspond to specific events control in the control level. With respect to the control level, the level consists of information about events that corresponds to the task authorization, which fulfills both the execution for a specific task and communication between the workflow level and the data level. Finally, the data level consists plenty of processes of data that related to a specific task. The process of multi-level context-aware data integration is illustrated as Fig.3, in which both state transitions and communications among the levels are fulfilled by some specific functions in order to fulfill data integrating based on dynamic material demands. And the functions are defined as following to illustrate the process of data integrating control:

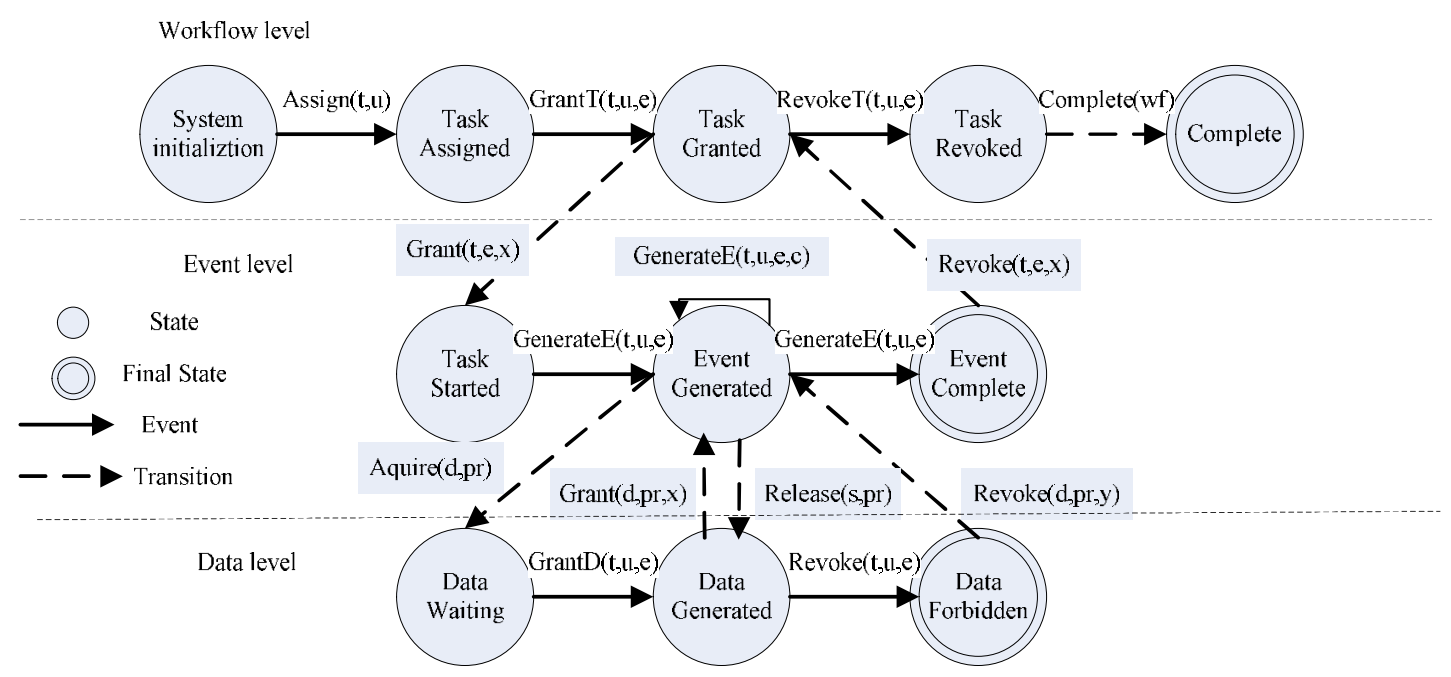

Fig.3. The Multi-level context-aware control model

For all functions $t \in T, e \in E, u \in U, d \in D, p r \in P R, c \in C$ and $x \in S(t)$.

$\mathrm{N}(\mathrm{t})=\mathrm{u}$ means that the user $\mathrm{u}$ can only execute the task $\mathrm{t}$, which in turn $\mathrm{t}$ is executed by $\mathrm{u}$.

$\mathrm{C}(\mathrm{u}) \in \mathrm{T}$ expresses that all tasks that the user $\mathrm{u}$ can execute.

$\mathrm{P}(\mathrm{d}) \in \mathrm{PR}$ expresses that all rights related to data $\mathrm{d}$.

$\operatorname{Assign}(\mathrm{t}, \mathrm{u})$ : $\mathrm{t}$ is assigned to $\mathrm{u}, \mathrm{t} \in \mathrm{C}(\mathrm{u})$ and $\mathrm{N}(\mathrm{t})=\mathrm{u}$.

GrantT(t,u,e): if $D(t)$ is executed and e as the input parameter for the specific $t$ is generated then $u$ is permitted to execute $t$. And the result of $\operatorname{GrantT}(\mathrm{t}, \mathrm{u}, \mathrm{e})$ is recorded in the $\mathrm{HU}(\mathrm{u})$ through the function Grant $(\mathrm{t}, \mathrm{e}, \mathrm{x})$, and $\mathrm{e} \in \mathrm{IE}(\mathrm{t})$.

RevokeT(t,u,e): when the $t$ is revoked all rights related to the $t$ for $u$ is revoked. And e as the output parameter is generated. And the result of RevokeT $(\mathrm{t}, \mathrm{u}, \mathrm{e})$ is recorded in the HU(u) through the function Revoke(t,e,x), and e $\in \mathrm{OE}(\mathrm{t})$.

GenerateE(t,u,e,c):the $\mathrm{e}$ is generated in the process of executing $\mathrm{t}$ and the result of GenerateE(t,u,e,c) that takes into account the contextual information is recorded in HT(t) through the function generate $(\mathrm{e}, \mathrm{x})$.

$\operatorname{GrantD}(\mathrm{t}, \mathrm{u}, \mathrm{e})$ : if the e as a request related to $\mathrm{d}$ is generated and ta belonging to TA permits e's operation then $\mathrm{u}$ is granted specific privilege rights for $\mathrm{d}$. And the result of the function is recorded in the $\mathrm{HU}(\mathrm{u})$ through the function $\operatorname{Grant}(\mathrm{d}, \mathrm{pr}, \mathrm{x}), \mathrm{e}=$ acquire $(\mathrm{d}, \mathrm{pr}) \in \mathrm{IE}(\mathrm{t}),(\mathrm{d}, \mathrm{pr}) \in \mathrm{F}$ that expresses that all matching relationships related to t between D set and PR set. 
RevokeD(t,u,e): when the e is revoked all rights related to the $\mathrm{t}$ is revoked for $\mathrm{u}$. And the result of $\operatorname{RevokeD}(\mathrm{t}, \mathrm{u}, \mathrm{e})$ is recorded in the $\mathrm{HU}(\mathrm{u})$ through the function $\operatorname{Revoke}(\mathrm{d}, \mathrm{pr}, \mathrm{x})$, and $\mathrm{e}=$ release $(\mathrm{d}, \mathrm{pr}) \in$ $\mathrm{IE}(\mathrm{t})$.

During the run time of data integrating, the multi-level context-aware model as a monitor manages the process of data integrating control from the viewpoint of task, event and data respectively at different levels.

\section{Case Study}

A material data control process is a base of successor application, by which we can make the material trace operation associated with equipment information, technology information, demand information and some base attribute information, as illustrated in Fig.4.

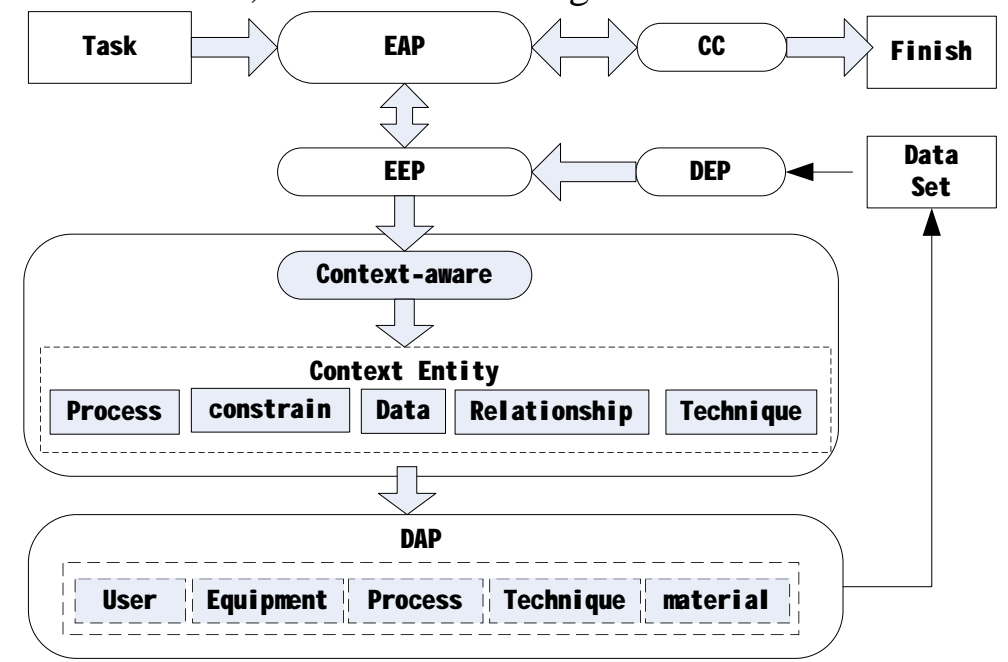

Fig.4. Multi-level data integrating control process

In Fig.4 the meaning of EAP is event assignment point, EEP is event execution point, DAP is data assignment point, DEP is data execution point, $\mathrm{CC}$ is checking point. A task execution consists of many state transitions triggered by specific events.

\section{Acknowledgments}

This work is supported by the Project which is supported by National Science \& Technology Pillar Program in the Twelfth Five-Year Plan Period (2013BAF02B03).

\section{References}

[1] F.Qiu, H.Wang, J.Li. Research And Development On Management Information System Of The Multiple-Sort \& Small-Batch Materials In Lean Production, Journal of Wuhan University of Technology,vol.28(2008), p.117

[2] G. Neto, A. Gomes, J. Castro and S. Sampaio, Integrating Activity Theory And Organizational Modeling For Context Of Use Analysis, Proceedings of the 2005 Latin American conference on Human-computer interaction, CLIHC 2005, p.301

[3] H.Lin, Y.Fan, C.Wu. Research Of Integrated Planning And Scheduling Based On Hierarchy Scheduling Model, Computer Integrated Manufacturing Systems, vol.8(2009),p.602

[4] W.vanderAalst, Business Alignment.Using Process Mining As A Tool For Delta Analysis, Journal of Requirements Engineering, vol.10(2008),p.198

[5] MARK T, ELMORE. Dynamic Data Fusion Using An Ontology-Based Software Agent System. Proceedings of the World Multi conference on Systemics. Cybernetics and Informatics, 2003, p.6 